$132(47 \%)$ did not attend. This was a highly significant difference $(P<0.01)$ and suggests that there is a great deal of reluctance for people to become associated with psychiatric hospitals.

The drive towards community-based care in the form of community health teams we would hope to be an important factor in eliminating this stigma by making the services more accessible and less intimidating for the people who may require their help.

In addition, patient access to medical records may have an important part to play in de-stigmatising psychiatry. Psychiatrists could take this opportunity to improve doctor/patient communication and show that psychiatry is not the secretive and sinister profession it is often portrayed to be.

T. N. Evans

Cefn Coed Hospital

A. IWANCZYK

Cockett, Swansea SA20GM

\section{Observation of the suicidal in-patient}

\section{DEAR SIRS}

There is substantial psychiatric literature concerning the assessment of suicidal risk. There is little empirical assessment of observation of the suicidal in-patient. It has been suggested that a code of practice might be formulated (Morgan, 1988). We felt it might be helpful to construct a picture of day-to-day clinical practice on a nation-wide basis.

A postal questionnaire concerning this subject received a disappointing response rate $(55 \%)$ which precludes meaningful statistical analysis. We feel, however, that the findings merit some discussion. The vast majority of units who responded had a written policy concerning observation. Two-thirds utilised constant surveillance of the patient. In the majority of units the level of observation was a multidisciplinary decision. In four, nursing staff alone made this decision.

A clear majority of respondents felt that a controlled trial of observation would be unethical. We would suggest that this probably reflects a high degree of confidence in this regime.

Our experience is that patients find this practice intrusive and often resent it. Conversely, relatives appear grateful for it. We do wonder, however, given the loss of privacy involved, whether there is a case for its being included as a treatment requiring informed consent or a second opinion from a psychiatrist approved under Part IV of the Mental Health Act 1983.

The final issue concerns the prison system. Despite calls for prison reform, there have so far been no moves to implement this regime for the suicidal patient within the prison system. The obvious difficulty here is the resource implications for over- stretched prison hospitals. Given that the option is a form of seclusion ("unfurnished accomodation") we would suggest that this issue should be urgently addressed.

Reaside Clinic

N. M. J. KENNEDY

Birmingham B45 $9 B E$

Lyndon Clinic, Hobs Meadow

N.S. BrowN

Solihull, West Midlands B92 8PW

\section{Reference}

Morgan H. G. (ed) (1981) Proceedings of Conference on the Clinical Management of Suicide Risk. London: The Royal Society of Medicine, Chapterhouse Codex.

\section{Air freshener perfumes}

DEAR SIRS

Over the last 12 years, I have collected 51 cases who appear to be adversely affected by the presence of so-called 'fresh' perfumes in their environment. They present with symptoms of tiredness, nausea, headache and mild derealisation. A minor subgroup suffer irritation of the nose, chest or eyes and three of the cases were severely disturbed emotionally, with tearfulness and inability to cope. One young man was contemplating suicide. Signs of fine tremor, intention tremor, nystagmus and an unsteady Rombergs test were present in most (Lawson, 1985). In all cases, symptoms resolved completely when they cleared sources of 'fresh' odourants from their environment. None of the cases went on to develop anxiety or depression needing treatment in its own right.

'Fresh' perfumes, like traditional perfumes, are a mixture of many different odourants, but a greater proportion of the constituents are synthetic in the case of the 'fresh' variety. There is also the possibility that a new agent - the 'Malodour Counteractant' discovered by A. Schleppink in 1968 and developed by Monsanto, has a specific mode of action in blocking the exchange of protons, which is a leading feature of unpleasant odourants (Schleppink, 1981).

It is thought that fresheners act by stimulating the olfactory nerve so strongly that unwanted odours are not perceived. The theoretical consequences of strong stimulation of the olfactory nerve are interesting. There is no doubt that the major input is to the rhinencephalon, now termed the limbic lobe, and thought to mediate emotion. There are also connections between the olfactory nerve and the hypothalamus and the caudate nucleus. There is therefore some theoretical basis for the observed effect on emotions and on muscle tone. Further corroboration of the likelihood of perfumes affecting mood comes from the work of Dodd, who has used perfumes to induce beneficial mood changes (Dodd \& Van Toller, 1983). 